\title{
On $\phi$-Absorbing Primary Elements in Lattice Modules
}

\author{
Sachin Ballal and Vilas Kharat \\ Department of Mathematics, Savitribai Phule Pune University, Pune 411 007, India \\ Correspondence should be addressed to Sachin Ballal; ballalshyam@gmail.com
}

Received 18 December 2014; Accepted 31 March 2015

Academic Editor: Andrei V. Kelarev

Copyright (c) 2015 S. Ballal and V. Kharat. This is an open access article distributed under the Creative Commons Attribution License, which permits unrestricted use, distribution, and reproduction in any medium, provided the original work is properly cited.

Let $L$ be a $C$-lattice and let $M$ be a lattice module over $L$. Let $\phi: M \rightarrow M$ be a function. A proper element $P \in M$ is said to be $\phi$-absorbing primary if, for $x_{1}, x_{2}, \ldots, x_{n} \in L$ and $N \in M, x_{1} x_{2} \cdots x_{n} N \leq P$ and $x_{1} x_{2} \cdots x_{n} N \not \varnothing \phi(P)$ together imply $x_{1} x_{2} \cdots x_{n} \leq\left(P: 1_{M}\right)$ or $x_{1} x_{2} \cdots x_{i-1} x_{i+1} \cdots x_{n} N \leq \sqrt[M]{P}$, for some $i \in\{1,2, \ldots, n\}$. We study some basic properties of $\phi$-absorbing primary elements. Also, various generalizations of prime and primary elements in multiplicative lattices and lattice modules as $\phi$-absorbing elements and $\phi$-absorbing primary elements are unified.

\section{Introduction}

A lattice $L$ is said to be complete, if, for any subset $S$ of $L$, we have $\vee S, \wedge S \in L$. Since every complete lattice is bounded, $1_{L}$ (or 1 ) denotes the greatest element and $0_{L}$ (or 0 ) denotes the smallest element of $L$. A complete lattice $L$ is said to be a multiplicative lattice, if there is a defined binary operation "." called multiplication on $L$ satisfying the following conditions:

(1) $a \cdot b=b \cdot a$, for all $a, b \in L$,

(2) $a \cdot(b \cdot c)=(a \cdot b) \cdot c$, for all $a, b, c \in L$,

(3) $a \cdot\left(\vee_{\alpha} b_{\alpha}\right)=\vee_{\alpha}\left(a \cdot b_{\alpha}\right)$, for all $a, b_{\alpha} \in L$,

(4) $a \cdot 1=a$, for all $a \in L$.

Henceforth, $a \cdot b$ will be simply denoted by $a b$.

An element $p \neq 1$ of a multiplicative lattice $L$ is said to be prime if $a b \leq p$ implies either $a \leq p$ or $b \leq p$, for $a, b \in L$. Radical of an element $a \in L$ is denoted by $\sqrt{a}$ and is defined as $\sqrt{a}=\vee\left\{x \in L \mid x^{n} \leq a\right.$, for some $\left.n \in \mathbb{Z}^{+}\right\}$.

An element $c$ of a complete lattice $L$ is said to be compact if $c \leq \vee_{\alpha} a_{\alpha}$ implies $c \leq \vee_{i=1}^{n} a_{\alpha_{i}}$, where $n \in \mathbb{Z}^{+}$. The set of all compact elements of a lattice $L$ is denoted by $L_{*}$. By a C-lattice we mean a multiplicative lattice $L$ with a multiplicatively closed set $S$ of compact elements which generates $L$ under join.

A complete lattice $M$ is said to be a lattice module over a multiplicative lattice $L$, if there is a multiplication between elements of $M$ and $L$, denoted by $a N$ for $a \in L$ and $N \in M$, which satisfies the following properties:

(1) $(a b) N=a(b N)$,

(2) $\left(\mathrm{V}_{\alpha} a_{\alpha}\right)\left(\mathrm{V}_{\beta} N_{\beta}\right)=\mathrm{V}_{\alpha, \beta}\left(a_{\alpha} N_{\beta}\right)$,

(3) $1_{L} N=N$,

(4) $0_{L} N=0_{M}$, for all $a, b, a_{\alpha} \in L$ and for all $N, N_{\beta} \in M$,

where $1_{M}$ denotes the greatest element of $M$ and $0_{M}$ denotes the smallest element of $M$.

For $N \in M$ and $a \in L$, denote $(N: a)=\vee\{X \in M$ : $a X \leq N\}$. For $a, b \in L,(a: b)=\vee\{x \in L: b x \leq a\}$ and for $A, B \in M,(A: B)=\vee\{x \in L: x B \leq A\}$. For $N \in M$, $\sqrt{N}=\vee\left\{x \in L: x^{n} 1_{M} \leq N\right\}$ for some positive integer $n$ and it is also denoted by $\sqrt{\left(N: 1_{M}\right)}$. For $N \in M$, we define $\sqrt[M]{N}=\sqrt{\left(N: 1_{M}\right)} 1_{M}$. An element $N \in M$ is said to be weak join principal if it satisfies the following identity $a \vee\left(0_{M}: N\right)=$ $(a N: N)$ for all $a \in L$.

A lattice module $M$ over a multiplicative lattice $L$ is called a multiplication lattice module if for $N \in M$ there exists an element $a \in L$ such that $N=a 1_{M}$.

An element $N \neq 1_{M}$ in $M$ is said to be prime if $a X \leq N$ implies $X \leq N$ or $a 1_{M} \leq N$, that is, $a \leq\left(N: 1_{M}\right)$ for every $a \in L$ and $X \in M$.

An element $N \in M$ is called compact if $N \leq \mathrm{V}_{\alpha} A_{\alpha}$ implies $N \leq A_{\alpha 1} \vee A_{\alpha 2} \vee \cdots \vee A_{\alpha n}$ for some $\alpha_{1}, \alpha_{2}, \ldots, \alpha_{n}$. If each element of $M$ is a join of principal (compact) elements of 
$M$, then $M$ is called principally generated lattice (compactly generated lattice).

Çallialp et al. [1] studied the concepts of weakly prime and almost prime elements in multiplicative lattices as extensions of, respectively, weakly prime and almost prime ideals in commutative rings. An element $p \neq 1$ in $L$ is said to be weakly prime if $0 \neq a b \leq p$ implies either $a \leq p$ or $b \leq p$ and almost prime if $a b \leq p$ and $a b \notin p^{2}$ implies either $a \leq p$ or $b \leq p$ for $a, b \in L$. In [2], the authors generalized these concepts, respectively, to weakly primary and almost primary elements in multiplicative lattices. A proper element $p \in L$ is said to be weakly primary if $0 \neq a b \leq p$ implies either $a \leq p$ or $b \leq \sqrt{p}$ and almost primary if $a b \leq p$ and $a b \notin p^{2}$ implies either $a \leq p$ or $b \leq \sqrt{p}$ for $a, b \in L$.

The concept of prime elements in multiplicative lattices is further generalised to 2-absorbing and weakly 2-absorbing elements in multiplicative lattices by Jayaram et al. [3]. An element $q<1$ in $L$ is said to be 2-absorbing if $a b c \leq q$ implies either $a b \leq q$ or $b c \leq q$ or $a c \leq q$ and is said to be weakly 2-absorbing element if $0 \neq a b c \leq q$ implies either $a b \leq q$ or $b c \leq q$ or $a c \leq q$, for $a, b, c \in L$. Joshi and Ballal [4] defined the concept of $n$-prime elements in multiplicative lattices as a generalization of prime elements. An element $p<1$ of a multiplicative lattice $L$ is said to be an $n$-prime if we can express it as meet of at most $n$ primes, where $n$ is a positive integer.

In [5], the authors defined the concepts of $n$-absorbing, weakly $n$-absorbing, and $n$-almost $n$-absorbing elements in multiplicative lattices as generalizations of, respectively, 2absorbing, weakly 2-absorbing, and almost prime elements, where $n \geq 2$. An element $p<1$ of a multiplicative lattice $L$ is called $n$-absorbing if $x_{1} x_{2} x_{3} \cdots x_{n+1} \leq p$ implies $x_{1} x_{2} \cdots x_{i-1} x_{i+1} \cdots x_{n+1} \leq p$ and called weakly $n$-absorbing if, for $x_{1}, x_{2}, x_{3}, \ldots, x_{n+1} \in L, 0 \neq x_{1} x_{2} x_{3} \cdots x_{n+1} \leq$ $p$ implies $x_{1} x_{2} \cdots x_{i-1} x_{i+1} \cdots x_{n+1} \leq p, i \in\{1,2, \ldots, n\}$, and $x_{1}, x_{2}, x_{3}, \ldots, x_{n+1} \in L$. An element $p<1$ of a multiplicative lattice $L$ is called $n$-almost $n$-absorbing if $x_{1} x_{2} x_{3} \cdots x_{n+1} \leq p$ and $x_{1} x_{2} x_{3} \cdots x_{n+1} \not p^{n}$ together imply $x_{1} x_{2} \cdots x_{i-1} x_{i+1} \cdots x_{n+1} \leq p$ for $i \in\{1,2, \ldots, n\}$, $x_{1}, x_{2}, x_{3}, \ldots, x_{n+1} \in L$.

Manjarekar and Bingi [6] unified the theory of generalizations of prime and primary elements in multiplicative lattice as $\phi$-prime and $\phi$-primary elements. Let $\phi: L \rightarrow L$ be a function. A proper element $p \in L$ is said to be $\phi$-prime if $a b \leq p$ and $a b \not \phi(p)$ implies either $a \leq p$ or $b \leq p$ and it is said to be $\phi$-primary if $a b \leq p$ and $a b \not \phi(p)$ implies either $a \leq p$ or $b \leq \sqrt{p}$, for $a, b \in L$.

As a generalization of primary ideals in commutative rings, Badawi et al. [7] introduced the concept of 2-absorbing primary ideals. In this paper, we extend the various generalizations of prime ideals and primary ideals in commutative rings to lattice modules. Also, we unify various generalizations of prime and primary elements in multiplicative lattices and lattice modules, respectively, as $\phi$-absorbing elements and $\phi$-absorbing primary elements.

For basic concepts and terminologies of lattice modules, one may refer to [8-11] and for multiplicative lattices, one may refer to [12-14].

\section{2. $\phi$-Absorbing Primary Elements}

We introduce the concepts of $\phi$-absorbing elements and $\phi$-absorbing primary elements in lattice modules which generalizes, respectively, the concepts of prime and primary elements in multiplicative lattices and lattice modules (see [1$3,5,6])$.

Essentially, we have the following definitions, where $M$ is a lattice module over a multiplicative lattice $L$ and $n \geq 1$.

Definition 1. A proper element $P \in M$ is said to be $n$ absorbing if $x_{1} x_{2} \cdots x_{n} N \leq P$ implies $x_{1} x_{2} \cdots x_{n} \leq\left(P: 1_{M}\right)$ or $x_{1} x_{2} \cdots x_{i-1} x_{i+1} \cdots x_{n} N \leq P$ for $i \in\{1,2, \ldots, n\}$, where $x_{1}, x_{2}, \ldots, x_{n} \in L$ and $N \in M$.

Definition 2. Let $\phi: M \rightarrow M$ be a function. A proper element $P \in M$ is said to be $\phi$-absorbing if $x_{1} x_{2} \cdots x_{n} N \leq P$ and $x_{1} x_{2} \cdots x_{n} N \not \subset(P)$ together imply $x_{1} x_{2} \cdots x_{n} \leq(P$ : $\left.1_{M}\right)$ or $x_{1} x_{2} \cdots x_{i-1} x_{i+1} \cdots x_{n} N \leq P$ for $i \in\{1,2, \ldots, n\}$, where $x_{1}, x_{2}, \ldots, x_{n} \in L$ and $N \in M$.

Definition 3. A proper element $P \in M$ is said to be $n$ absorbing primary if $x_{1} x_{2} \cdots x_{n} N \leq P$ implies $x_{1} x_{2} \cdots x_{n} \leq$ $\left(P: 1_{M}\right)$ or $x_{1} x_{2} \cdots x_{i-1} x_{i+1} \cdots x_{n} N \leq \sqrt[M]{P}$ for $i \in\{1,2, \ldots, n\}$, where $x_{1}, x_{2}, \ldots, x_{n} \in L$ and $N \in M$.

Definition 4. Let $\phi: M \rightarrow M$ be a function. A proper element $P \in M$ is said to be $\phi$-absorbing primary if $x_{1} x_{2} \cdots x_{n} N \leq P$ and $x_{1} x_{2} \cdots x_{n} N \not \phi(P)$ together imply $x_{1} x_{2} \cdots x_{n} \leq\left(P: 1_{M}\right)$ or $x_{1} x_{2} \cdots x_{i-1} x_{i+1} \cdots x_{n} N \leq \sqrt[M]{P}$ for $i \in\{1,2, \ldots, n\}$, where $x_{1}, x_{2}, \ldots, x_{n} \in L$ and $N \in M$.

Let $M$ be a lattice module over a multiplicative lattice $L$. For a map $\phi_{\alpha}: M \rightarrow M$, we have the following.

(1) $\phi_{0}: \phi(P)=0$ defines weakly $n$-absorbing primary elements of $M$.

(2) $\phi_{2}: \phi(P)=\left(P: 1_{M}\right) P$ defines almost $n$-absorbing primary elements of $M$.

(3) $\phi_{m+1}(n \geq 1): \phi(P)=\left(P: 1_{M}\right)^{m} P$ defines $m$-almost $n$-absorbing primary elements of $M$.

(4) $\phi_{\omega}: \phi(P)=\wedge_{m=1}^{\infty}\left(P: 1_{M}\right)^{m} P$ defines $\omega$-absorbing primary elements of $M$.

Remark 5. It follows immediately from the definition that any $n$-absorbing primary element of $M$ is a $\phi$-absorbing primary. However, the converse does not necessarily holds. In fact, we have the following theorem in which the converse is true under certain condition.

Theorem 6. Let $M$ be a lattice module over a $C$-lattice $L$. Then every $\phi$-absorbing primary element $P \in M$ with $\left(P: 1_{M}\right)^{n} P \nless$ $\phi(P)$ is n-absorbing primary.

Proof. Suppose that $\left(P: 1_{M}\right)^{n} P \not \Varangle \phi(P)$ and $x_{1} x_{2} \cdots x_{n} N \leq$ $P$, for $x_{1}, x_{2}, \ldots, x_{n} \in L, N \in M$. If $x_{1} x_{2} \cdots x_{n} N \not \phi(P)$, then as $P$ is $\phi$-absorbing primary, we have $x_{1} x_{2} \cdots x_{n} \leq\left(P: 1_{M}\right)$ or $x_{1} x_{2} \cdots x_{i-1} x_{i+1} \cdots x_{n} N \leq \sqrt[M]{P}$ for some $i \in\{1,2, \ldots, n\}$, which concludes that $P$ is $n$-absorbing primary. 
Now, suppose that $x_{1} x_{2} \cdots x_{n} N \leq \phi(P)$. We assume that $x_{1} x_{2} \cdots x_{n-k}\left(P: 1_{M}\right)^{k} N \leq \phi(P)$, for all $k \in\{1,2, \ldots, n-$ 1\}. If $x_{1} x_{2} \cdots x_{n-k}\left(P: 1_{M}\right)^{k} N \$ \phi(P)$, then there exists $a_{1}, a_{2}, \ldots, a_{k} \leq\left(P: 1_{M}\right)$ such that $x_{1} x_{2} \cdots x_{n-k} a_{1} a_{2} \cdots a_{k} N \not$ $\phi(P)$. Hence, $x_{1} x_{2} \cdots x_{n-k}\left(x_{n-k+1} \vee a_{1}\right)\left(x_{n-k+2} \vee a_{2}\right) \cdots\left(x_{n} \vee\right.$ $\left.a_{k}\right) N \leq P$ and $x_{1} x_{2} \cdots x_{n-k}\left(x_{n-k+1} \vee a_{1}\right)\left(x_{n-k+2} \vee a_{2}\right) \cdots\left(x_{n} \vee\right.$ $\left.a_{k}\right) N \not \phi(P)$.

Since $p$ is $\phi$-absorbing primary, $x_{1} x_{2} \cdots x_{n-k}\left(x_{n-k+1} \vee\right.$ $\left.a_{1}\right) \cdots\left(x_{n} \vee a_{k}\right) \leq\left(P: 1_{M}\right)$ or $x_{1} x_{2} \cdots x_{i-1} x_{i+1} \cdots$ $x_{n-k}\left(x_{n-k+1} \vee a_{1}\right)\left(x_{n-k+2} \vee a_{2}\right) \cdots\left(x_{n} \vee a_{k}\right) N \leq \sqrt[M]{P}$ for some $i \in\{1,2, \ldots, n-1\}$ and so $x_{1} x_{2} \cdots x_{i-1} x_{i+1}$ $\cdots x_{n-k} x_{n-k+1} x_{n-k+2} \cdots x_{n} N \leq \sqrt[M]{P}$ for some $i \in\{1,2, \ldots, n-$ $1\}$ or $x_{1} x_{2} \cdots x_{n} \leq\left(P: 1_{M}\right)$.

Similarly, we assume that, for $\left\{i_{1}, i_{2}, \ldots, i_{n-k}\right\} \subseteq$ $\{1,2, \ldots, n\}, 1 \leq k \leq n, x_{i_{1}} x_{i_{2}} \cdots x_{i_{n-k}}\left(P: 1_{M}\right)^{k} N \leq \phi(P)$. Also, we assume that $x_{i_{1}} x_{i_{2}} \cdots x_{i_{n-k}}\left(P: 1_{M}\right)^{k} P \leq \phi(P)$, $1 \leq k \leq n$, because if $x_{i_{1}} x_{i_{2}} \cdots x_{i_{n-k}}\left(P: 1_{M}\right)^{k} P \not \phi(P)$, then $x_{i_{1}} x_{i_{2}} \cdots x_{i_{n-k}} a_{1} a_{2} \cdots a_{k} P \quad \$ \quad \phi(P)$, where $a_{1} a_{2} a_{3} \cdots a_{k} \leq\left(P: 1_{M}\right)$ and so $x_{1} x_{2} \cdots x_{n-k}\left(x_{n-k+1} \vee\right.$ $\left.a_{1}\right)\left(x_{n-k+2} \vee a_{2}\right) \cdots\left(x_{n} \vee a_{k}\right)(N \vee P) \leq \sqrt[M-k]{P}$ and $x_{1} x_{2} \cdots x_{n-k}\left(x_{n-k+1} \vee a_{1}\right)\left(x_{n-k+2} \vee a_{2}\right) \cdots\left(x_{n} \vee a_{k}\right)(N \vee P) \not$ $\phi(P)$.

Now, since $P$ is $\phi$-absorbing primary, $x_{1} x_{2} \cdots x_{n} \leq$ $\left(P: 1_{M}\right)$ or $x_{1} x_{2} \cdots x_{i-1} x_{i+1} \cdots x_{n} N \leq \sqrt[M]{P}$, for some $i \epsilon$ $\{1,2, \ldots, n-1\}$.

Also, $\left(P \quad: \quad 1_{M}\right)^{n} P \quad \not \quad \phi(P)$ implies that there exist $a_{1}, a_{2}, \ldots, a_{n} \leq\left(P: 1_{M}\right)$ with $a_{1} a_{2} \cdots a_{n} P \$ \phi(P)$. We have $\left(x_{1} \vee a_{1}\right)\left(x_{2} \vee a_{2}\right) \cdots\left(x_{n} \vee a_{n}\right)(N \vee P) \leq P$ and $\left(x_{1} \vee a_{1}\right)\left(x_{2} \vee\right.$ $\left.a_{2}\right) \cdots\left(x_{n} \vee a_{n}\right)(N \vee P) \$ \phi(P)$. Now, $P$ is $\phi$-absorbing primary; therefore, $x_{1} x_{2} \cdots x_{i-1} x_{i+1} \cdots x_{n-k} x_{n-k+1} x_{n-k+2} \cdots x_{n} N \leq P$, for some $i \in\{1,2, \ldots, n-1\}$ or $x_{1} x_{2} \cdots x_{n} \leq\left(P: 1_{M}\right)$ and consequently $P$ is $\phi$-absorbing primary.

From the above theorem, it follows that if $P$ is a $\phi$-primary element of $M$ that is not $n$-absorbing primary, then ( $P$ : $\left.1_{M}\right)^{n} P \leq \phi(P)$.

Corollary 7. Let $M$ be a lattice module over a C-lattice L. If $P \in M$ is weakly n-absorbing primary that is not n-absorbing primary, then $\left(P: 1_{M}\right)^{n} P=0$.

Theorem 8. Let $M$ be a multiplication lattice module over $C$ lattice $L$ and let $P \in M$. Then the following holds.

(1) Let $\psi_{1}, \psi_{2}: M \rightarrow M$ be two functions with $\psi_{1} \leq \psi_{2}$, that is, $\psi_{1}(N) \leq \psi_{2}(N)$ for each $N \in M$. Then $P$ is $\psi_{2}$-absorbing primary if it is $\psi_{1}$-absorbing primary.

(2) Consider the following statements.

(a) $P$ is n-absorbing primary.

(b) $P$ is weaklyn-absorbing primary.

(c) $P$ is $\omega$-absorbing primary.

(d) $P$ is m-almost n-absorbing primary.

(e) $P$ is almost $n$-absorbing primary.

Then $(\mathrm{a}) \Rightarrow(\mathrm{b}) \Rightarrow(\mathrm{c}) \Rightarrow(\mathrm{d}) \Rightarrow$ (e).

(3) $P$ is $\omega$-absorbing primary if and only if it is $m$-almost $n$-absorbing primary for $m \geq 1$.
Proof. (1) Suppose that $P \in M$ is $\psi_{1}$-absorbing primary and also suppose that $x_{1} x_{2} \cdots x_{n} N \leq P$ and $x_{1} x_{2} \cdots x_{n} N \not \psi_{2}(P)$ for $x_{1}, x_{2}, \ldots, x_{n} \in L$ and $N \in M$. Since $\psi_{1}(N) \leq \psi_{2}(N)$ for each $N \in M$, we have $x_{1} x_{2} \cdots x_{n} N \nless \psi_{1}(P)$. It follows from the fact $x_{1} x_{2} \cdots x_{n} N \leq P, x_{1} x_{2} \cdots x_{n} N \not \psi_{1}(P)$, and $P$ is $\psi_{1}$-absorbing primary that $x_{1} x_{2} \cdots x_{n} \leq\left(P: 1_{M}\right)$ or $x_{1} x_{2} \cdots x_{i-1} x_{i+1} \cdots x_{n} N \leq \sqrt[M]{P}$ for some $i \in\{1,2, \ldots, n\}$ and so, $P$ is $\psi_{2}$-absorbing primary.

(2) By definition, every $n$-absorbing primary element is weakly $n$-absorbing primary and therefore $(\mathrm{a}) \Rightarrow$ (b) holds.

(b) $\Rightarrow$ (c) Suppose that $P$ is weakly $n$-absorbing primary and also suppose that $x_{1} x_{2} \cdots x_{n} N \leq P$ and $x_{1} x_{2} \cdots x_{n} N \$$ $\wedge_{n=1}^{\infty}\left(P: 1_{M}\right)^{n} P$, for $x_{1}, x_{2}, \ldots, x_{n} \in L$ and $N \in M$. Then $x_{1} x_{2} \cdots x_{n} N \neq 0_{M}$. By the assumption, $x_{1} x_{2} \cdots x_{n} \leq\left(P: 1_{M}\right)$ or $x_{1} x_{2} \cdots x_{i-1} x_{i+1} \cdots x_{n} N \leq \sqrt[M]{P}$ for some $i \in\{1,2, \ldots, n\}$ and so, $P$ is $\omega$-absorbing primary.

(c) $\Rightarrow$ (d) Suppose that $P$ is $\omega$-absorbing primary and also suppose that $x_{1} x_{2} \cdots x_{n} N \leq P$ and $x_{1} x_{2} \cdots x_{n} N \not(P$ : $\left.1_{M}\right)^{m} P$ for $x_{1}, x_{2}, \ldots, x_{n} \in L, N \in M$, and $m \geq 1$. Then $x_{1} x_{2} \cdots x_{n} N \leq P$ and $x_{1} x_{2} \cdots x_{n} N \$ \wedge_{m=1}^{\infty}\left(P: 1_{M}\right)^{m} P$. Since $P$ is $\omega$-absorbing primary, it follows that $x_{1} x_{2} \cdots x_{n} \leq$ $\left(P: 1_{M}\right)$ or $x_{1} x_{2} \cdots x_{i-1} x_{i+1} \cdots x_{n} N \leq \sqrt[M]{P}$ for some $i \epsilon$ $\{1,2, \ldots, n\}$ and so, $P$ is $m$-almost $n$-absorbing primary.

The statement (e) is a particular case of the statement (d) for $m=1$ and therefore (d) $\Rightarrow$ (e) holds.

(3) Suppose that $P \in M$ is $m$-almost $n$-absorbing primary for $m \geq 1$ and also suppose that $x_{1} x_{2} \cdots x_{n} N \leq P$ and $x_{1} x_{2} \cdots x_{n} N \$ \wedge_{m=1}^{\infty}\left(P: 1_{M}\right)^{m} P$ for $x_{1}, x_{2}, \ldots, x_{n} \in L$ and $N \in M$. Then $x_{1} x_{2} \cdots x_{m} N \leq P$ and $x_{1} x_{2} \cdots x_{n} N \not$ $\left(P: 1_{M}\right)^{m} P$ for some $m \geq 1$. Since $P$ is $m$-almost $n$ absorbing primary, we have $x_{1} x_{2} \cdots x_{n} \leq\left(P: 1_{M}\right)$ or $x_{1} x_{2} \cdots x_{i-1} x_{i+1} \cdots x_{n} N \leq \sqrt[M]{P}$ for some $i \in\{1,2, \ldots, n\}$. Consequently, $P$ is $\omega$-absorbing primary.

The converse follows from $(c) \Rightarrow(d)$.

Corollary 9. Let $M$ be a lattice module over a C-lattice $L$. If $P \in M$ is $\phi$-absorbing primary, where $\phi \leq \phi_{n+2}$, then $P$ is $\omega$ absorbing primary.

Proof. Suppose that $P \in M$ is $\phi$-absorbing primary. If $P$ is $n$-absorbing primary, then, by Theorem 8 , it is $\omega$-absorbing primary. Now, if $P$ is not $n$-absorbing primary, then, by Theorem 6, $\left(P: 1_{M}\right)^{n} P \leq \phi(P) \leq \phi_{n+2}(P)=\left(P: 1_{M}\right)^{n+1} P$. Consequently, $\phi(P)=\left(P: 1_{M}\right)^{k} P$, for each $k \geq n$. And, by Theorem $8(3), P$ is $\omega$-absorbing primary.

Lemma 10. Let $L$ be a multiplicative lattice. If $p \in L$ is $\phi$ absorbing primary with $\phi(\sqrt{p})=\sqrt{\phi(p)}$, then $\sqrt{p}$ is also $\phi$ absorbing.

Proof. (I) Suppose that $x_{1} x_{2} \cdots x_{n+1} \leq \sqrt{p}$ and $x_{1} x_{2} \cdots x_{n+1} \nless \phi(\sqrt{p})=\sqrt{\phi(p)}$, for $x_{1}, x_{2}, \ldots, x_{n+1} \in L$. If $x_{1} x_{2} \cdots x_{i-1} x_{i+1} \cdots x_{n+1} \$ \sqrt{p}$ for $i \in\{1,2, \ldots, n\}$, then we have $\left(x_{1} x_{2} \cdots x_{i-1} x_{i+1} \cdots x_{n+1}\right)^{s} \nless \neq p$ for any positive integer $s$ and for $i \in\{1,2, \ldots, n\}$.

(II) Now, since $x_{1} x_{2} \cdots x_{n+1} \leq \sqrt{p}$, there exists a positive integer $k$ such that $\left(x_{1} x_{2} \cdots x_{n+1}\right)^{k}=x_{1}^{k} x_{2}^{k} \cdots x_{n+1}^{k} \leq p$. 
(III) As $x_{1} x_{2} \cdots x_{n+1} \nless \sqrt{\phi(p)}$, we have $x_{1}^{t} x_{2}^{t} \cdots x_{n+1}^{t} \not$ $\phi(p)$ for a positive integer $t$.

From the assumption and (I), (II), and (III), it follows that $\left(x_{1} x_{2} \cdots x_{n}\right)^{k}=x_{1}^{k} x_{2}^{k} \cdots x_{n}^{k} \leq \sqrt{p}$. Consequently, $x_{1} x_{2} \cdots x_{n} \leq \sqrt{\sqrt{p}}$ and therefore $\sqrt{p}$ is $\phi$-absorbing primary.

It is easy to observe that if $\left(L_{1}, \wedge_{1}, \vee_{1},{ }^{\circ}\right)$ and $\left(L_{2}, \wedge_{2}\right.$, $\left.\vee_{2}, \circ_{2}\right)$ are multiplicative lattices then $L_{1} \times L_{2}$ is also a multiplicative lattice with componentwise meet, join, and multiplication.

Also, if $M_{1}$ and $M_{2}$ are lattice modules over multiplicative lattices $L_{1}$ and $L_{2}$, respectively, then $M_{1} \times M_{2}$ is a lattice module over $L_{1} \times L_{2}$ with componentwise meet, join, and multiplication given by $(a, b)\left(N_{1}, N_{2}\right)=\left(a N_{1}, b N_{2}\right)$, where $(a, b) \in L_{1} \times L_{2}$ and $\left(N_{1}, N_{2}\right) \in M_{1} \times M_{2}$.

Lemma 11. Let $M=M_{1} \times M_{2}$ and $L=L_{1} \times L_{2}$, where $M_{i}$ is a lattice module over $C$-lattice $L_{i}$, for $i=1,2$. Then $\left(\left(P_{1}\right.\right.$ : $\left.\left.1_{M_{1}}\right),\left(P_{2}: 1_{M_{2}}\right)\right)=\left(\left(P_{1}, P_{2}\right):\left(1_{M_{1}}, 1_{M_{2}}\right)\right)$, for $P_{1} \in M_{1}$ and $P_{2} \in M_{2}$.

Proof. Let $(x, y) \in L_{*}$. Now, $(x, y) \leq\left(\left(P_{1}: 1_{M_{1}}\right),\left(P_{2}: 1_{M_{2}}\right)\right)$ :

$$
\begin{aligned}
& \Leftrightarrow x \leq\left(P_{1}: 1_{M_{1}}\right) \text { and } y \leq\left(P_{2}: 1_{M_{2}}\right), \\
& \Leftrightarrow x 1_{M_{1}} \leq P_{1} \text { and } y 1_{M_{2}} \leq P_{2}, \\
& \Leftrightarrow(x, y)\left(1_{M_{1}}, 1_{M_{2}}\right)=\left(x 1_{M_{1}}, y 1_{M_{2}}\right) \leq\left(P_{1}, P_{2}\right), \\
& \Leftrightarrow(x, y) \leq\left(P_{1}, P_{2}\right):\left(1_{M_{1}}, 1_{M_{2}}\right) .
\end{aligned}
$$

Lemma 12. Let $M=M_{1} \times M_{2}$ and $L=L_{1} \times L_{2}$, where $M_{i}$ is a lattice module over C-lattice $L_{i}$, for $i=1,2$. Then $\sqrt[M]{\left(P_{1}, P_{2}\right)}=$ $\left(\sqrt[M_{1}]{P_{1}}, \sqrt[M_{2}]{P_{2}}\right)$, for $P_{1} \in M_{1}$ and $P_{2} \in M_{2}$.

Proof. Let $\left(N_{1}, N_{2}\right) \in M_{*}$ with $\left(N_{1}, N_{2}\right) \leq \sqrt[M]{\left(P_{1}, P_{2}\right)}=$ $\sqrt{\left(P_{1}, P_{2}\right):\left(1_{M_{1}}, 1_{M_{2}}\right)}\left(1_{M_{1}}, 1_{M_{2}}\right)$. Then $\left(N_{1}, N_{2}\right) \leq$ $(a, b)\left(1_{M_{1}}, 1_{M_{2}}\right)$, where $(a, b) \leq \sqrt{\left(P_{1}, P_{2}\right):\left(1_{M_{1}}, 1_{M_{2}}\right)}$.

Now, $(a, b) \leq \sqrt{\left(P_{1}, P_{2}\right):\left(1_{M_{1}}, 1_{M_{2}}\right)} \Rightarrow(a, b)^{n} \leq\left(P_{1}, P_{2}\right)$ : $\left(1_{M_{1}}, 1_{M_{2}}\right)$ for a positive integer $n$ :

$$
\begin{aligned}
& \Rightarrow a^{n} \leq\left(P_{1}: 1_{M_{1}}\right) \text { and } b^{n} \leq\left(P_{2}: 1_{M_{2}}\right) \text { (by Lemma 11), } \\
& \Rightarrow(a, b) \leq\left(\sqrt{\left(P_{1}: 1_{M_{1}}\right)}, \sqrt{\left(P_{2}: 1_{M_{2}}\right)}\right) \\
& \Leftrightarrow(a, b)\left(1_{M_{1}}, 1_{M_{2}}\right) \leq\left(\sqrt{\left(P_{1}: 1_{M_{1}}\right)}, \sqrt{\left(P_{2}: 1_{M_{2}}\right)}\right)\left(1_{M_{1}},\right. \\
& \left.1_{M_{2}}\right)=\left(\sqrt{\left(P_{1}: 1_{M_{1}}\right)} 1_{M_{1}}, \sqrt{\left(P_{2}: 1_{M_{2}}\right)} 1_{M_{2}}\right) \\
& \Leftrightarrow(a, b)\left(1_{M_{1}}, 1_{M_{2}}\right) \leq\left(\sqrt[M_{1}]{P_{1}}, \sqrt[M_{2} 2]{P_{2}}\right) .
\end{aligned}
$$

Consequently,

$$
\sqrt[M]{\left(P_{1}, P_{2}\right)} \leq\left(\sqrt[M]{P_{1}}, \sqrt[M]{P_{2}}\right)
$$
$\left.\sqrt[M_{2}]{P_{2}}\right)$ :

$$
\begin{aligned}
& \Rightarrow S_{1} \leq \sqrt[\sqrt[M]{1} /]{P_{1}} \text { and } S_{2} \leq \sqrt[M_{2}]{P_{2}} ; \\
& \Rightarrow S_{1} \leq \sqrt{\left(P_{1}: 1_{M_{1}}\right)} 1_{M_{1}} \text { and } S_{2} \leq \sqrt{\left(P_{2}: 1_{M_{2}}\right)} 1_{M_{2}} ; \\
& \Rightarrow S_{1} \leq x 1_{M_{1}} \text { and } S_{2} \leq y 1_{M_{2}} \text { for some } x, y \in L \text { with } \\
& x \leq \sqrt{\left(P_{1}: 1_{M_{1}}\right)} \text { and } y \leq \sqrt{\left(P_{2}: 1_{M_{2}}\right)} ; \\
& \Rightarrow x^{t} \leq\left(P_{1}: 1_{M_{1}}\right) \text { and } y^{r} \leq\left(P_{2}: 1_{M_{2}}\right) \text { for some } \\
& \text { positive integers } t \text { and } r .
\end{aligned}
$$

Choose $k=t+r$. Then $x^{k} \leq\left(P_{1}: 1_{M_{1}}\right)$ and $y^{k} \leq\left(P_{2}: 1_{M_{2}}\right)$ :

$$
\begin{aligned}
& \Rightarrow(x, y)^{k}=\left(x^{k}, y^{k}\right) \leq\left(\left(P_{1}: 1_{M_{1}}\right),\left(P_{2}: 1_{M_{2}}\right)\right) ; \\
& \Rightarrow(x, y) \leq \sqrt{\left(\left(P_{1}: 1_{M_{1}}\right),\left(P_{2}: 1_{M_{2}}\right)\right)} ; \\
& \Rightarrow(x, y)\left(1_{M_{1}}, 1_{M_{2}}\right) \leq \sqrt{\left(\left(P_{1}: 1_{M_{1}}\right),\left(P_{2}: 1_{M_{2}}\right)\right)}\left(1_{M_{1}},\right. \\
& \left.1_{M_{2}}\right) ; \quad \quad\left(S_{1}, S_{2}\right) \quad \leq \quad(x, y)\left(1_{M_{1}}, 1_{M_{2}}\right) \\
& \Rightarrow \quad \leq \\
& \sqrt{\left(\left(P_{1}: 1_{M_{1}}\right),\left(P_{2}: 1_{M_{2}}\right)\right)}\left(1_{M_{1}}, 1_{M_{2}}\right)=\sqrt[M]{\left(P_{1}, P_{2}\right)} .
\end{aligned}
$$

Consequently,

$$
\left(\sqrt[M_{1}]{P_{1}}, \sqrt[M_{2}]{P_{2}}\right) \leq \sqrt[M]{\left(P_{1}, P_{2}\right)}
$$

From (I) and (II), result follows.

Theorem 13. Let $M=M_{1} \times M_{2}$ and $L=L_{1} \times L_{2}$, where $M_{i}$ is a lattice module over C-lattice $L_{i}$, for $i=1,2$, and let $\phi: M \rightarrow$ $M$ be a function. If $P_{1} \in M_{1}$ is a weakly n-absorbing primary such that $\left(0,1_{M_{2}}\right) \leq \phi\left(P_{1}, 1_{M_{2}}\right)$, then $\left(P_{1}, 1_{M_{2}}\right) \in M_{1} \times M_{2}$ is $\phi$-absorbing primary.

Proof. Let $\left(a_{1}, b_{1}\right),\left(a_{2}, b_{2}\right), \ldots,\left(a_{n}, b_{n}\right) \in L$ and $\left(N_{1}, N_{2}\right) \in M$ be such that $\left(a_{1}, b_{1}\right)\left(a_{2}, b_{2}\right) \cdots\left(a_{n}, b_{n}\right)\left(N_{1}, N_{2}\right)=$ $\left(a_{1} a_{2} \cdots a_{2} N_{1}, b_{1} b_{2} \cdots b_{2} N_{2}\right) \leq\left(P_{1}, 1_{M_{2}}\right)$ and $\left(a_{1}, b_{1}\right)\left(a_{2}, b_{2}\right)$ $\cdots\left(a_{n}, b_{n}\right)\left(N_{1}, N_{2}\right)=\left(a_{1} a_{2} \cdots a_{n} N_{1}, b_{1} b_{2} \cdots b_{n} N_{2}\right) \not \phi\left(P_{1}\right.$, $\left.1_{M_{2}}\right)$.

Since $\left(0,1_{M_{2}}\right) \leq \phi\left(P_{1}, 1_{M_{2}}\right)$, we have $\left(a_{1}, b_{1}\right)\left(a_{2}, b_{2}\right) \ldots$ $\left(a_{n}, b_{n}\right)\left(N_{1}, N_{2}\right)=\left(a_{1} a_{2} \cdots a_{n} N_{1}, b_{1} b_{2} \cdots b_{n} N_{2}\right) \not\left(0,1_{M_{2}}\right)$ and so $0 \neq a_{1} a_{2} \cdots a_{n} N_{1} \leq P_{1}$. Since $P_{1} \in M_{1}$ is weakly $n$-absorbing primary, we have $a_{1} a_{2} \cdots a_{n} \leq$ $\left(P_{1}: 1_{M_{1}}\right)$ or $a_{1} a_{2} \cdots a_{i-1} a_{i+1} \cdots a_{n} N_{1} \leq \sqrt[M_{1}]{P_{1}}$, for some $i \in\{1,2, \ldots, n\}$. This implies that $\left(a_{1}, b_{1}\right)\left(a_{2}\right.$, $\left.b_{2}\right) \cdots\left(a_{n}, b_{n}\right)=\left(a_{1} a_{2} \cdots a_{n}, b_{1} b_{2} \cdots b_{n}\right) \leq\left(\left(P_{1}: 1_{M_{1}}\right)\right.$, $\left.\left(1_{M_{2}}: 1_{M_{2}}\right)\right)=\left(\left(P_{1}, 1_{M_{2}}\right):\left(1_{M_{1}}, 1_{M_{2}}\right)\right)$ or $\left(a_{1}, b_{1}\right)\left(a_{2}, b_{2}\right)$ $\cdots\left(a_{i-1}, b_{i-1}\right)\left(a_{i+1}, b_{i+1}\right) \cdots\left(a_{n}, b_{n}\right)\left(N_{1}, N_{2}\right) \leq\left(\sqrt[M_{1}]{P_{1}}, 1_{M_{2}}\right)=$ $\sqrt[M]{\left(P_{1}, 1_{M_{2}}\right)}$ for some $i \in\{1,2, \ldots, n\}$, by Lemmas 11 and 12. Therefore, $\left(P_{1}, 1_{M_{2}}\right)$ is $\phi$-absorbing primary element of $M_{1} \times M_{2}$.

Theorem 14. Let $M$ be a lattice module over a $C$-lattice $L$ and $P \in M$. Then $P$ is $\phi$-absorbing primary if and only if $\left(P \quad: \quad x_{1} x_{2} \cdots x_{n-1} N\right)=\left(P \quad: x_{1} x_{2} \cdots x_{n-1} 1_{M}\right)$ or $\left(P: x_{1} x_{2} \cdots x_{n-1} N\right)=\left(\phi(P): x_{1} x_{2} \cdots x_{n-1} N\right)$ or $(P$ : $\left.x_{1} x_{2} \cdots x_{n-1} N\right)=\left(\sqrt{P}: x_{1} x_{2} \cdots x_{i-1} x_{i+1} \cdots x_{n-1} N\right)$ for some $i \in\{1,2, \ldots, n-1\}$, for $x_{1}, x_{2}, \ldots, x_{n-1} \in L$ and $N \in M$ with $x_{1} x_{2} \cdots x_{n-1} N \$ \sqrt[M]{P}$. 
Proof. Suppose that $P \in M$ is $\phi$-absorbing primary and $x_{1} x_{2} \cdots x_{n-1} N \$ \sqrt[M]{P}$, for $x_{1}, x_{2}, \ldots, x_{n-1} \in L$ and $N \in M$. Let $r \in L_{*}$ be such that $r \leq\left(P: x_{1} x_{2} \cdots x_{n-1} N\right)$ which essentially implies that $x_{1} x_{2} \cdots x_{n-1} r N \leq P$.

We have the following two cases.

Case 1. If $r x_{1} x_{2} \cdots x_{n-1} N \not \$ \phi(P)$, then as $P$ is $\phi$ absorbing primary, we have $x_{1} x_{2} \cdots x_{n-1} r \leq\left(P: 1_{M}\right)$ or $x_{1} x_{2} \cdots x_{i-1} x_{i+1} \cdots x_{n-1} r N \leq \sqrt[M]{P}$ for some $i \in\{1,2, \ldots, n-$ $1\}$. Therefore, $r \leq\left(P: x_{1} x_{2} \cdots x_{n-1} 1_{M}\right)$ or $r \leq(\sqrt[M]{P}$ : $\left.x_{1} x_{2} \cdots x_{i-1} x_{i+1} \cdots x_{n-1} N\right)$ for some $i \in\{1,2, \ldots, n-1\}$.

Case 2. If $r x_{1} x_{2} \cdots x_{n-1} N \leq \phi(P)$, then $r \leq(\phi(P)$ : $\left.x_{1} x_{2} \cdots x_{n-1} N\right)$. So $\left(P: x_{1} x_{2} \cdots x_{n-1} N\right) \leq(\phi(P)$ : $\left.x_{1} x_{2} \cdots x_{n-1} N\right)$.

Now, from Cases 1 and 2 , it follows that $(P$ : $\left.x_{1} x_{2} \cdots x_{n-1} N\right)=\left(P: x_{1} x_{2} \cdots x_{n-1} 1_{M}\right)$ or $\left(P: x_{1} x_{2} \cdots\right.$ $\left.x_{n-1} N\right)=\left(\phi(P): x_{1} x_{2} \cdots x_{n-1} N\right)$ or $\left(P: x_{1} x_{2} \cdots x_{n-1} N\right)=$ $\left(\sqrt[M]{P}: x_{1} x_{2} \cdots x_{i-1} x_{i+1} \cdots x_{n-1} N\right)$ for some $i \in\{1,2, \ldots, n-1\}$.

Conversely, suppose that $x_{1} x_{2} \cdots x_{n} N \leq P$ and $x_{1} x_{2} \cdots x_{n} N \not \phi(P)$, for $x_{1}, x_{2}, \ldots, x_{n} \in L$ and $N \in M$. If $x_{1} x_{2} \cdots x_{n-1} N \leq \sqrt[M]{P}$, then the result is obvious. So, suppose that $x_{1} x_{2} \cdots x_{n-1} N \$ \sqrt[M]{P}$.

Now, $\left(P: x_{1} x_{2} \cdots x_{n-1} N\right)=\left(P: x_{1} x_{2} \cdots x_{n-1} 1_{M}\right)$ or $\left(P: x_{1} x_{2} \cdots x_{n-1} N\right)=\left(\phi(P): x_{1} x_{2} \cdots x_{n-1} N\right)$ or $(P:$ $\left.x_{1} x_{2} \cdots x_{n-1} N\right)=\left(\sqrt[M]{P}: x_{1} x_{2} \cdots x_{i-1} x_{i+1} \cdots x_{n-1} N\right)$ for some $i \in\{1,2, \ldots, n-1\}$. Since $x_{1} x_{2} \cdots x_{n} N \leq P$, we have $x_{n} \leq$ $\left(p: x_{1} x_{2} \cdots x_{n-1} N\right)$. But $x_{n} \not\left(\phi(P): x_{1} x_{2} \cdots x_{n-1} N\right)$ and so $x_{1} x_{2} \cdots x_{n-1} x_{n} \leq\left(P: 1_{M}\right)$ or $x_{1} x_{2} \cdots x_{i-1} x_{i+1} \cdots x_{n} N \leq \sqrt[M]{P}$ for some $i \in\{1,2, \ldots, n\}$. Consequently, $P$ is $\phi$-absorbing primary.

Theorem 15. Let $M=M_{1} \times M_{2} \times \cdots \times M_{n}$ and $L=L_{1} \times L_{2} \times$ $\cdots \times L_{n}$, where each $M_{i}$ is a compactly generated lattice module over a $C$-lattice $L_{i}$, for $i \in\{1,2, \ldots, n\}$. Let $\psi: M \rightarrow M$ such that $\psi(P)=\left(\psi_{1}\left(P_{1}\right), \psi_{2}\left(P_{2}\right), \ldots, \psi_{n}\left(P_{n}\right)\right)$, where $P_{i} \in M_{i}$, $\psi_{i}: M_{i} \rightarrow M_{i}, i \in\{1,2, \ldots, n\}$, and $P=\left(P_{1}, P_{2}, \ldots, P_{n}\right)$ is $\psi-$ absorbing primary. Then $P_{i}$ is a $\psi_{i}$-absorbing primary element of $M_{i}$, for each $i$ with $P_{i} \neq 1_{M_{i}}$.

Proof. Let $P_{i} \neq 1_{M_{i}}, N_{i} \in M_{i}$, and $x_{1}, x_{2}, \ldots, x_{n} \in L_{i}$ be such that $x_{1} x_{2} \cdots x_{n} N_{i} \leq P_{i}$ and $x_{1} x_{2} \cdots x_{n} N_{i} \not \psi_{i}\left(P_{i}\right)$. Thus $\left(1, \ldots, 1, x_{1}, 1, \ldots, 1\right)\left(1, \ldots, 1, x_{2}, 1, \ldots, 1\right) \cdots\left(1, \ldots, 1, x_{n}, 1\right.$, $\ldots, 1)\left(0, \ldots, 0, N_{i}, 0, \ldots, 0\right)=\left(0, \ldots, 0, x_{1} x_{2} \cdots x_{n} N_{i}\right.$, $0, \ldots, 0) \leq P$, and $\left(0, \ldots, 0, x_{1} x_{2} \cdots x_{n} N_{i}, 0, \ldots, 0\right) \nless \psi(P)$. As $P$ is $\psi$-absorbing primary, $\left(1,1, \ldots, x_{1} x_{2} \cdots x_{n}, 0, \ldots, 0\right) \leq$ $\left(P: 1_{M}\right)$ or $\left(1,1, \ldots, x_{1} x_{2} \cdots x_{i-1} x_{i+1} \cdots x_{n} N_{i}, 0, \ldots, 0\right) \leq$ $\sqrt[M]{P}$. Now, by Lemmas 11 and 12 , we have $x_{1} x_{2} \cdots x_{n} \leq\left(P_{i}\right.$ : $1_{M_{i}}$ ) or $x_{1} x_{2} \cdots x_{i-1} x_{i+1} \cdots x_{n} N_{i} \leq \sqrt[M]{P_{i}}$ for $i \in\{1,2, \ldots, n\}$ and consequently, $P_{i}$ is $\psi_{i}$-absorbing primary element of $M_{i}$, for each $i$.

Corollary 16. Let $M=M_{1} \times M_{2} \times \cdots \times M_{n}$ and $L=L_{1} \times L_{2} \times$ $\cdots \times L_{n}$, where each $M_{i}$ is a compactly generated lattice module over a C-lattice $L_{i}$, for $i \in\{1,2, \ldots, n\}$. If $P=\left(P_{1}, P_{2}, \ldots, P_{n}\right) \in$ $M$ is $\phi_{m}$-absorbing primary, where $P_{i} \in M_{i}$, then $P_{i} \in M_{i}$ is $\phi_{m}$-absorbing primary with $P_{i} \neq 1_{M_{i}}(n, m \geq 2)$.
Proof. We have $\phi_{m}(P)=\left(P \quad: \quad 1_{M}\right)^{m-1} P=\left(\left(P_{1}\right.\right.$ : $\left.\left.1_{M_{1}}\right)^{m-1} P_{1},\left(P_{2} \quad: \quad 1_{M_{2}}\right)^{m-1} P_{2}, \ldots,\left(P_{n}: 1_{M_{n}}\right)^{m-1} P_{n}\right)=$ $\left(\phi_{m}\left(P_{1}\right), \phi_{m}\left(P_{2}\right), \ldots, \phi_{m}\left(P_{n}\right)\right)$ and the result follows from Theorem 15 .

Theorem 17. Let $M$ be a lattice module over a C-lattice $L$. Suppose that $a 1_{M} \in M$ is a weak join principal element with $a 1_{M} \neq 1_{M}$ and $\left(0_{M}: a 1_{M}\right) \leq \sqrt{a}$. Then $a 1_{M}$ is $m$-almost $n$-absorbing primary, $m \geq 1$, if and only if it is $n$-absorbing primary.

Proof. Suppose that $a 1_{M} \in M$ is $m$-almost $n$-absorbing primary and $x_{1} x_{2} \cdots x_{n} N \leq a 1_{M}$, for $x_{1}, x_{2}, \ldots, x_{n} \in L$ and $N \in M$. If $x_{1} x_{2} \cdots x_{n} N \not \subset\left(a 1_{M}: 1_{M}\right)^{m} a 1_{M}$, then $x_{1} x_{2} \cdots x_{n} \leq\left(a 1_{M}: 1_{M}\right)$ or $x_{1} x_{2} \cdots x_{i-1} x_{i+1} \cdots x_{n} N \leq$ $\sqrt[M]{a 1_{M}}$ for some $i \in\{1,2, \ldots, n\}$ and therefore $a 1_{M}$ is $n$ absorbing primary.

Now, suppose that $x_{1} x_{2} \cdots x_{n} N \leq\left(a 1_{M}: 1_{M}\right)^{m} a 1_{M}$. Since $x_{1} x_{2} \cdots x_{n} N \leq a 1_{M}$, we have $\left(x_{1} \vee a\right) x_{2} \cdots x_{n} N \leq a 1_{M}$.

If $\left(x_{1} \vee a\right) x_{2} \cdots x_{n} N \not\left(a 1_{M}: 1_{M}\right)^{m} a 1_{M}$, then it follows from the fact $\left(x_{1} \vee a\right) x_{2} \cdots x_{n} N \leq a 1_{M},\left(x_{1} \vee a\right) x_{2} \cdots x_{n} N \not$ $\left(a 1_{M}: 1_{M}\right)^{m} a 1_{M}$, and $a 1_{M}$ is $m$-almost $n$-absorbing primary that $x_{1} x_{2} \cdots x_{n} \leq\left(a 1_{M}: 1_{M}\right)$ or $x_{1} x_{2} \cdots x_{i-1} x_{i+1} \cdots x_{n} N \leq$ $\sqrt[M]{a 1_{M}}$ for some $i \in\{1,2, \ldots, n\}$ and we are done.

So assume that $\left(x_{1} \vee a\right) x_{2} \cdots x_{n} N \leq\left(a 1_{M}: 1_{M}\right)^{m} a 1_{M}$. Then as $x_{1} x_{2} \cdots x_{n} N \leq\left(a 1_{M}: 1_{M}\right)^{n} a 1_{M}$, we have $a x_{2} \cdots x_{n} N \leq\left(a 1_{M}: 1_{M}\right)^{n} a 1_{M}$. Next, $a x_{2} \cdots x_{n} N \leq\left(a 1_{M}\right.$ : $\left.1_{M}\right)^{n} a 1_{M}$ and $a 1_{M}$ is weak join principal; together they imply that $x_{2} \cdots x_{n} \leq\left(\left(a 1_{M}: 1_{M}\right)^{n} a 1_{M}: a 1_{M}\right)=\left(a 1_{M}\right.$ : $\left.1_{M}\right)^{n} \vee\left(0_{M}: a 1_{M}\right) \leq a \vee\left(0_{M}: a 1_{M}\right) \leq \sqrt{a}$. Consequently, $x_{2} \cdots x_{n} N \leq \sqrt{a} N \leq \sqrt{a} 1_{M}=\sqrt[M]{a 1_{M}}$ which implies that $a 1_{M}$ is $n$-absorbing primary.

The converse follows from Theorem $8(2)$.

Note. The results pertaining to $\phi$-absorbing elements are essentially the corollaries to the respective results of $\phi$ absorbing primary elements, as such the results of $\phi$ absorbing elements are the immediate consequences of results of $\phi$-absorbing primary elements in this paper.

\section{Conflict of Interests}

The authors declare that there is no conflict of interests regarding the publication of this paper.

\section{Acknowledgment}

This research work is an outcome of the project supported by Board of College and University Development, Savitribai Phule Pune University, Pune.

\section{References}

[1] F. Çallıalp, C. Jayaram, and Ü. Tekir, "Weakly prime elements in multiplicative lattices," Communications in Algebra, vol. 40, no. 8, pp. 2825-2840, 2012.

[2] S. B. Ballal, M. T. Gophane, and V. S. Kharat, "On weakly primary elements in multiplicative lattices," Southeast Asian Bulletin of Mathematics. In press. 
[3] C. Jayaram, Ü. Tekir, and E. Yetkin, "2-absorbing and weakly 2absorbing elements in multiplicative lattices," Communications in Algebra, vol. 42, no. 6, pp. 2338-2353, 2014.

[4] V. V. Joshi and S. B. Ballal, "A note on n-Baer multiplicative lattices," Southeast Asian Bulletin of Mathematics, vol. 39, pp. 6776, 2015.

[5] S. B. Ballal and V. S. Kharat, "On generalization of prime, weakly prime and almost prime elements in multiplicative lattices," International Journal of Algebra, vol. 8, no. 9, pp. 439-449, 2014.

[6] C. S. Manjarekar and A. V. Bingi, " $\emptyset$-prime and $\emptyset$-primary elements in multiplicative lattices," Algebra, vol. 2014, Article ID 890312, 7 pages, 2014.

[7] A. Badawi, Ü. Tekir, and E. Yetkin, "On 2-absorbing primary ideals in commutative rings," Bulletin of the Korean Mathematical Society, vol. 51, no. 4, pp. 1163-1173, 2014.

[8] E. A. Al-Khouja, "Maximal elements and prime elements in lattice modules," Damascus University for Basic Sciences, vol. 19, pp. 9-20, 2003.

[9] F. Callialp and Ü. Tekir, "Multiplication lattice modules," Iranian Journal of Science and Technology, vol. 35, no. 4, pp. 309-313, 2011.

[10] E. W. Johnson and J. A. Johnson, "Lattice modules over principal element domains," Communications in Algebra, vol. 31, no. 7, pp. 3505-3518, 2003.

[11] H. M. Nakkar and D. D. Anderson, "Associated and weakly associated prime elements and primary decomposition in lattice modules," Algebra Universalis, vol. 25, no. 2, pp. 196-209, 1988.

[12] F. Alarcon, D. D. Anderson, and C. Jayaram, "Some results on abstract commutative ideal theory," Periodica Mathematica Hungarica, vol. 30, no. 1, pp. 1-26, 1995.

[13] D. D. Anderson, "Abstract commutative ideal theory without chain condition," Algebra Universalis, vol. 6, no. 2, pp. 131-145, 1976.

[14] R. P. Dilworth, "Abstract commutative ideal theory," Pacific Journal of Mathematics, vol. 12, pp. 481-498, 1962. 


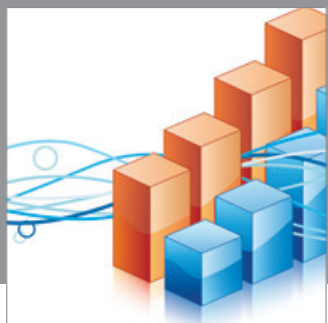

Advances in

Operations Research

mansans

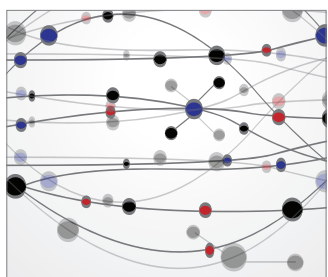

The Scientific World Journal
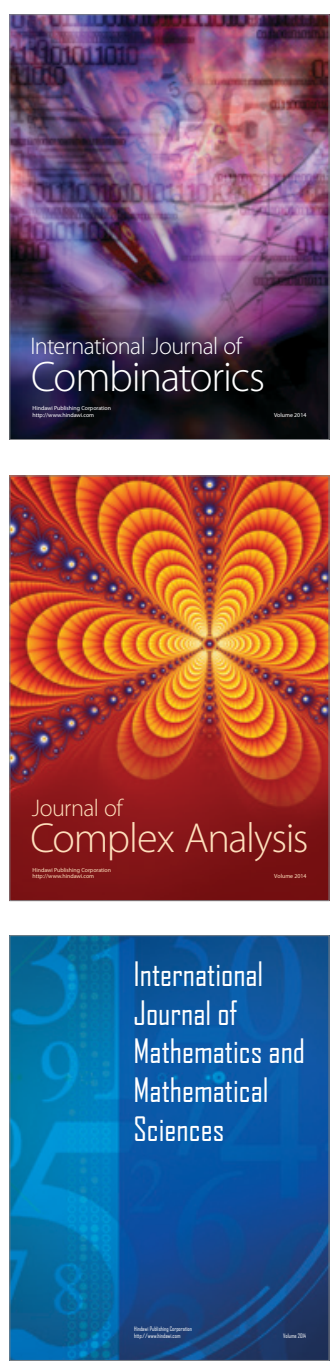
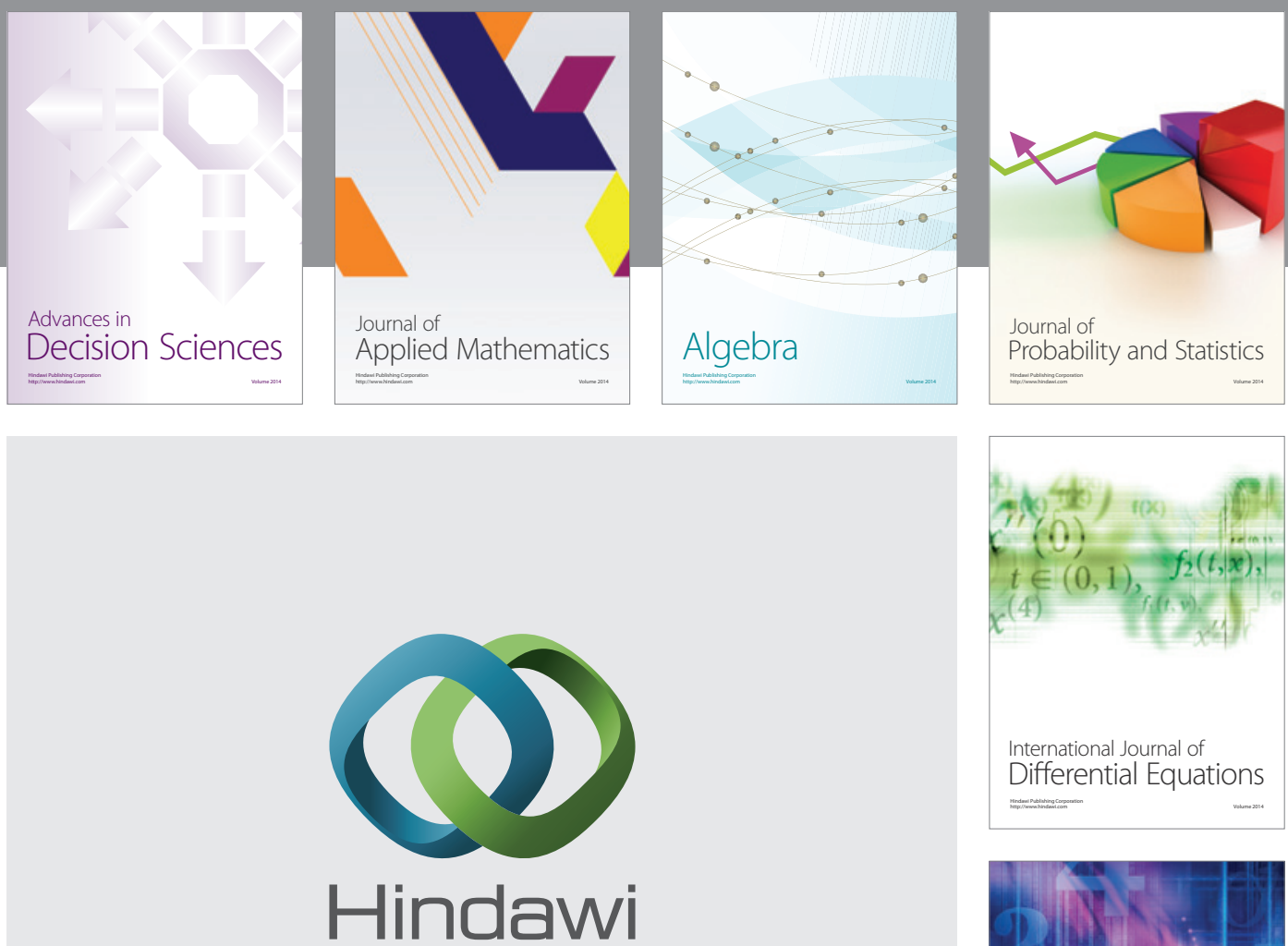

Submit your manuscripts at http://www.hindawi.com
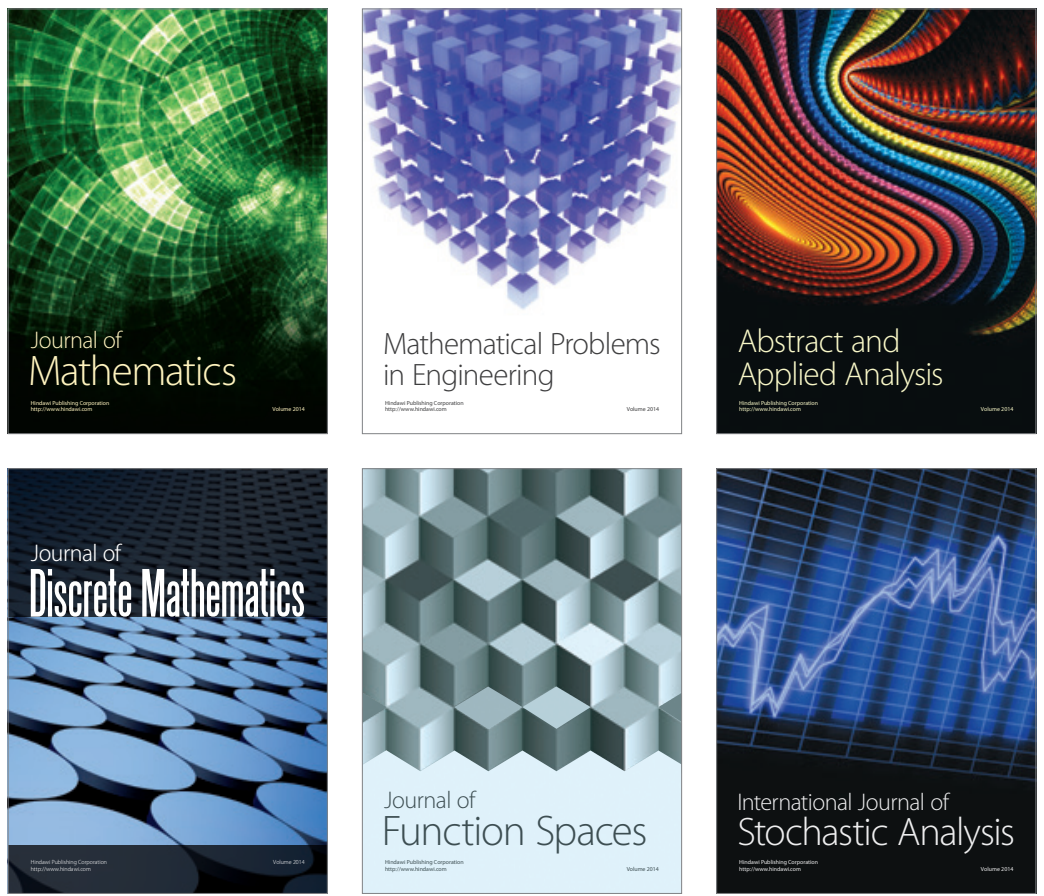

Journal of

Function Spaces

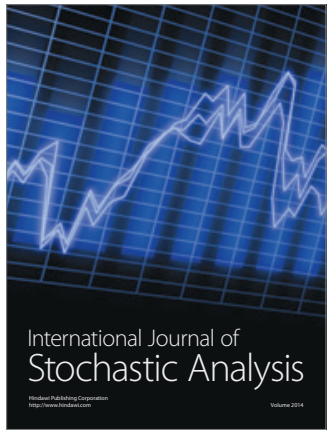

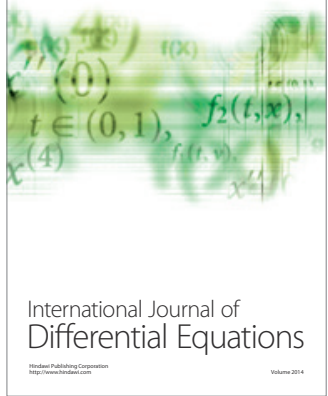
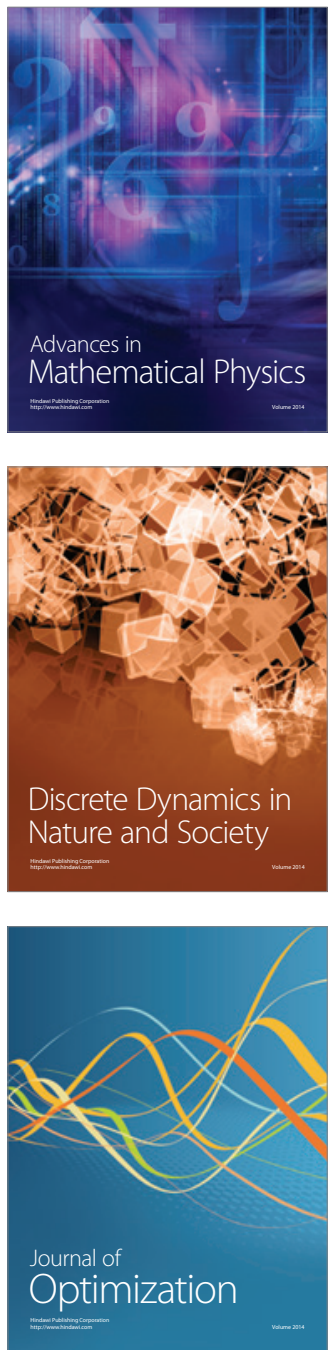